\title{
IAMJ
}

INTERNATIONAL

AYURVEDIC

MEDICAL JOURNAL

\section{VISHAHARA DRAVYAS IN MADANAPALA NIGHANTU - A REVIEW ARTICLE}

\author{
$\underline{\text { Haritha }}^{1}$, $\underline{\text { Ravikrishna }}^{2}, \underline{\text { Sreejith }}^{3}$, $\underline{\text { Chaithra S Hebbar }}^{4}$
}

${ }^{1}$ Post Graduate Scholar, ${ }^{2}$ Associate Professor, ${ }^{3}$ Assistant Professor, ${ }^{4}$ Professor and Head, Department of PG studies in Agadatantra, Sri Dharmasthala Manjunatheswara College of Ayurveda and Hospital, Kuthpady, Udupi-574118, Karnataka, India

Corresponding Author: harithaharidas005@gmail.com

\section{https://doi.org/10.46607/iamj1309122021}

(Published Online: December 2021)

Open Access

(C) International Ayurvedic Medical Journal, India

Article Received: 03/11//2021 - Peer Reviewed: 02/12/2021 - Accepted for Publication 04/12/2021

\section{Check for updates}

\section{ABSTRACT}

Background: Ayurveda is the science of life that deals with the protection of the health of the healthy and pacifying the disease of the diseased. Agada tantra is one of the branches of Ayurveda that deals with the signs and symptoms with treatment of visha of all types. Madanapala Nighantu is one of the ancient Nighantu written by King Madanapala. A total of 13 chapters are explained in Madanapala Nighantu and there are many vishahara dravyas explained throughout these chapters. Materials and Method: Here an attempt is made to collect and review the Vishahara dravya mentioned in the various vargas of Madanapala Nighantu with its botanical name, family and indication and is presented in the table. Observations and results: Among 13 Chapters of Madanapala Nighantu 105 dravyas are vishahara in nature. Conclusion: Either a single drug preparation or their combinations can give great results in the management of various poisoning.

Keywords: Agada tantra, Madanapala Nighantu, Visha, Vishahara.

\section{INTRODUCTION}

Agada tantra is the science that deals with the signs and symptoms and the management of all kinds of poisoning, various other poisons formed by the im- proper combination of substances or drugs and includes in detail explanation of mythology of origin of visha, visha pareeksha, various types of visha, guna 
of visha, visha vegas and their management. The reference of Agada tantra is available from the samhitha kala itself and hence it is found in various samhithas and other granthas. Visha is derived from the root word 'Vish' dhatu and ' $K$ ' pratyaya. ${ }^{[1]}$ That substance that creates vishada in creatures is considered as Visha. ${ }^{[2]}$ There are numerous Agada yogas as well as single drug preparations explained in Brihathrayis, laghutrayis and various agada tantra granthas. Nighantu refers to a tradition of Ayurvedic literature. Nighantu may be defined as a glossary containing synonymous groups, the names of the drugs, plants, animals, minerals or anything that is administered either as food or medicines, to the human body. Raja Nighantu explains the importance of Nighantu as "A physician without the knowledge of Nighantu, a scholar without the knowledge of grammar and a soldier without weapons, all these three are laughed at in this world". From this statement, it is clear that the knowledge of Nighantu is indispensable for a physician. ${ }^{[3]}$

Madanapala Nighantu was written by the King Madanapala of ' $t i k a$ ' dynasty during 1374 AD. Madanapala Nighantu is also famous as' Madanavi- noda'. A total of 13 chapters are explained in Madanapala Nighantu, in which there are 510 plant drugs, 56 mineral drugs, 11 animal drugs, 74 mamsa are explained and there are separate chapters such as paniyadi varga, Ikshuvadi varga, Dhanyakritannadi varga and Misraga varga. It was considered as the reference text for some of the nighantus such as Raja Nighantu and Bhavaprakasha Nighantu. King Narahari, the author of Raja Nighantu has been mentioned Madanapala Nighantu in his work. The remarkable feature of Madanapala Nighantu is that each chapter starts with some prayer on lord kirshna. There are various opinions regarding the author of Madanapala Nighantu. The introduction to some drugs such as Bhanga, Ahiphena, Jayapala, Kharjura, Amruthaphala etc. can be seen in Madanapala Nighantu. [4]

\section{MATERIALS AND METHODS}

The whole article is a literary review based on the Vishahara dravyas explained in Madanapala Nighantu. In this review, an attempt is made to collect all the vishahara dravyas mentioned in Madanapala Nighantu with its botanical name, family and indication, and are presented in respective vargas.

\section{OBSERVATIONS AND RESULTS}

Table 1: Vishahara dravyas in Abhayadi Varga with respective reference






\begin{tabular}{|c|c|c|c|c|c|}
\hline 16. & Katabhi & Clitoria ternatea Linn. & Fabaceae & Vishapaha & M.P.N.1/308 \\
\hline 17. & Sarapunkha & Tephrosia purpurea Linn. & Fabaceae & Vishapaha & M.P.N.1/314 \\
\hline 18. & Balamota & Sesbania aegyptiaca Pers. & Fabaceae & Visha & M.P.N.1/315 \\
\hline 19. & Arka dvaya & $\begin{array}{l}\text { Calotropis Procera (Ait) R.Br. } \\
\text { (Rakta arka) }\end{array}$ & Periplocaceae & Visha & M.P.N.1/323 \\
\hline 20. & & $\begin{array}{l}\text { Calotropis gigantean (Linn.) R.Br.ex. } \\
\text { Ait (Sweta arka) }\end{array}$ & Periplocaceae & Visha & M.P.N.1/323 \\
\hline
\end{tabular}

\section{*M.P. N- Madanapala Nighantu}

Table 2: Vishahara dravyas in Sunthyadi varga with respective reference

\begin{tabular}{|l|l|l|l|l|l|}
\hline Sr. No. & Drug Name & Botanical Name & Family & Indication & Reference \\
\hline 1. & $\begin{array}{l}\text { Cavya/ Gajapippali } \\
\text { (pushpa) }\end{array}$ & $\begin{array}{l}\text { Piper chaba } \text { Hunter. / Piper retrofractum } \\
\text { Vahl. }\end{array}$ & Piperaceae & $\begin{array}{l}\text { Gara vinasha- } \\
\text { na }\end{array}$ & M.P.N.2/19 \\
\hline
\end{tabular}

Table 3: Vishahara dravyas in Karpuradi Varga with respective reference

\begin{tabular}{|c|c|c|c|c|c|}
\hline $\begin{array}{l}\text { Sr. } \\
\text { No. }\end{array}$ & Drug Name & Botanical Name & Family & Indication & Reference \\
\hline 1. & Karpura & $\begin{array}{l}\text { Cinnamomum camphora (Linn.) Nees } \\
\text { Eberm }\end{array}$ & Lauraceae & Vishapaha & M.P.N.3/3 \\
\hline 2. & Kasturi & Moschus moschiferus Linn. & Cervidae & Visha & M.P.N.3/5 \\
\hline 3. & Candana & Santalum album Linn. & Santalaceae & Vishajith & M.P.N.3/9 \\
\hline 4. & Rakta candana & Pterocarpus santalinus Linn.f. & Fabaceae & Vishapaham & M.P.N.3/11 \\
\hline 5. & Jatipatri & Myristica fragrans Houtt. & Myristicaceae & Vishapaha & M.P.N.3/20 \\
\hline 6. & Sthula ela & Ammomum subulatum Roxb. & Scitaminaceae & Visha & M.P.N.3/26 \\
\hline 7. & Twak & Cinnamomum zeylanicum Blume. & Lauraceaea & Vishahara & M.P.N.3/28 \\
\hline 8. & Nagakesara & Mesua ferrea Linn. & Guttiferae & Vishapaham & M.P.N.3/30 \\
\hline 9. & Usira & Vetiveria zizanioides (Linn.) Nash. & Poaceae & Vishapaham & M.P.N.3/39 \\
\hline 10. & Rala & Shorea robusta Gaertn. F. & Dipterocarpaceae & Visha & M.P.N.3/51 \\
\hline 11. & Tagara & Valeriana wallichi D.C. & Valerianaceae & Vishapaham & M.P.N.3/63 \\
\hline 12. & \multirow[t]{2}{*}{ Nakhadvaya } & Capparis sepiaria Linn. (Himsra) & Capparidaceae & Vishapaham & M.P.N.3/67 \\
\hline 13. & & Capparis zeylanica Linn. (Grdhranakhi) & Capparidaceae & Vishapaham & M.P.N.3/67 \\
\hline 14. & Padmini & Nelumbium speciosum Willd. & Nymphaceae & Vishajith & M.P.N.3/73 \\
\hline 15. & Kamala & Nelumbo nucifera Linn. & Nymphaceae & Vishanashana & M.P.N.3/79 \\
\hline 16. & Vasuka & Osmanthus fragrans Lour. & Oleaceae & Visha & M.P.N.3/98 \\
\hline 17. & Kunda & Jasminum multiflorum (Burn.f.) Andr. & Oleaceae & Vishajith & M.P.N.3/99 \\
\hline 18. & Bhumandali & Cassia angustifolia Vahl. & Caesalpinaceae & Vishapaha & M.P.N.3/101 \\
\hline 19. & Maruvaka & Origanum majorana Linn. & Lamiaceae & Vrischikadi visha & M.P.N.3/108 \\
\hline 20. & \multirow[t]{3}{*}{ Barbari traya } & Ocimum basilicum Linn. (Vana tulasi) & Lamiaceae & Vishapaham & M.P.N.3/111 \\
\hline 21. & & $\begin{array}{l}\text { Ocimum gratissimum Linn. (Sveta Vana } \\
\text { tulasi) }\end{array}$ & Lamiaceae & Vishapaham & M.P.N.3/111 \\
\hline 22. & & $\begin{array}{l}\text { Ocimum kilmandscharicum Guerke. } \\
\text { (Karpura tulasi) }\end{array}$ & Lamiaceae & Vishapaham & M.P.N.3/111 \\
\hline
\end{tabular}

Table 4: Vishahara dravyas in Suvarnadi varga with respective reference 


\begin{tabular}{|c|c|c|c|c|c|}
\hline Sr. No. & Drug Name & Scientific Name & English name & Indication & Reference \\
\hline 1. & Suvarna & Aurum & Gold & Vishajith & M.P.N.4/4 \\
\hline 2. & Loha & Ferrum & Iron & Gara visha & M.P.N. 4/15 \\
\hline 3. & Mandura & Ferrous oxide & Iron rust & Gara visha & M.P.N. 4/16 \\
\hline 4. & Makshika & Copper and iron pyrite & Double pyrite & Visha & M.P.N. 4/24 \\
\hline 5. & Manashila & Arsenic dioxide & Realgar & Vishajith & M.P.N. 4/26 \\
\hline 6. & Haritala & Arsenic trioxide & Orpiment & Visham jayeth & M.P.N. 4/27 \\
\hline 7. & \multirow[t]{2}{*}{ Gairika dvaya } & Ferrous oxide (Gairika) & Ochre & Vishapaham & M.P.N. 4/29 \\
\hline 8. & & (Swarna gairika) & Ochre & Vishapaham & M.P.N. 4/29 \\
\hline 9. & \multirow[t]{2}{*}{ Tuttha dvaya } & Copper sulphate (Tuttha) & Blue vitriol & Vishapaham & M.P.N. 4/31 \\
\hline 10. & & (Sikhi kanda) & Blue vitriol & Vishapaham & M.P.N. 4/31 \\
\hline 11. & \multirow[t]{2}{*}{ Kasisa dvaya } & Ferrous sulphate (Dhatu kasisa) & Iron sulphate & visha & M.P.N. 4/33 \\
\hline 12. & & (Pushpa kasisa) & Iron sulphate & visha & M.P.N. 4/33 \\
\hline 13. & Hingula & Mercuric sulphate & Cinnabar & Visha & M.P.N. 4/34 \\
\hline 14. & Sindura & Lead oxide & Red lead & Vishapaham & M.P.N. 4/36 \\
\hline 15. & \multirow[t]{2}{*}{ Sphatika dvaya } & Potash alum (Spatika) & Alum & Visha & M.P.N. 4/47 \\
\hline 16. & & (Tuvari) & Alum & Visha & M.P.N. 4/47 \\
\hline 17. & Mukta & Pinctada vulgaris & Pearl & Vishanashana & M.P.N. 4/50 \\
\hline 18. & Chumbaka & Magnetite ore & Magnet & Vishapaha, garaapaha & M.P.N. 4/67 \\
\hline
\end{tabular}

- Pravala, mukta, manikya, suryakantamani, candrakantamani, gomeda, vajra, vaidurya, nila, garutmata vishapaha - M.P.N. 4/60,61

Table 5: Vishahara dravyas in Vatadi varga with respective reference

\begin{tabular}{|l|l|l|l|l|l|}
\hline Sr. No. & Drug Name & Botanical Name & Family & Indication & Reference \\
\hline 1. & Nadivriksha & Ficus retusa Linn. & Moraceae & Garanuth & M.P.N.5/12 \\
\hline 2. & Kakuba & Terminalia arjuna (Roxb.) W. \& A. & Combretaceae & Vishajith & M.P.N.5/14 \\
\hline 3. & Sisira & Albizia lebbeck (Linn.) Benth. & Mimosaceae & Visha & M.P.N.5/15 \\
\hline 4. & Hijjala & Barringtonia acutangula (Linn.) gartn. & Barringtoniaceae & Vishanashana & M.P.N.5/21 \\
\hline 5. & Shleshmataka & Cordia dichotoma Forst.f. & Boraginaceae & Visha & M.P.N.5/23 \\
\hline 6. & Sala & Shorea robusta Gaertn.F. & Dipterocarpaceae & Visha & M.P.N.5/27 \\
\hline 7. & Arimeda & Acacia farneciana (L.) Willd. & Mimosaceae & Visha & M.P.N.5/32 \\
\hline 8. & Babbula & Acacia nilotica (L.) & Mimosaceae & Visha & M.P.N.5/33 \\
\hline 9. & Inguda & Balanites roxburghii Planch. & Simaroubaceae & Visha & M.P.N.5/48 \\
\hline 10. & Katambhara & Albizia procera Benth. & Mimosaceae & Visha & M.P.N.5/49 \\
\hline 11. & Muska & Schrebera swietenoides Roxb. & Oleaceae & Visha & M.P.N.5/51 \\
\hline 12. & Tinthini & Trichodesma zeylanicum R.Br. & Boranginaceae & Vishapaha & M.P.N.5/66 \\
\hline
\end{tabular}

Table 6: Vishahara dravyas in Phaladi varga with respective reference

\begin{tabular}{|l|l|l|l|l|l|}
\hline Sr. No. & Drug Name & Botanical Name & Family & Indication & Reference \\
\hline 1. & Sevya & Pyrus malus Linn. & Rosaceae & Vishapaham & M.P.N.6/58 \\
\hline 2. & Vikantaka & Flacourtia ramontchi L. Herit. & Flacourtiaceae & Lootha vinashana & M.P.N.6/2 \\
\hline 3. & Kapittha patri & Feroniella oblate Swingle. & Rutaceae & Vishapaha & M.P.N.6/92 \\
\hline
\end{tabular}

Table 7: Vishahara dravyas in Saka varga with respective reference 


\begin{tabular}{|l|l|l|l|l|l|}
\hline Sr. No. & Drug Name & Botanical Name & Family & Indication & Reference \\
\hline 1. & Katutumbi & Lagenaria vulgaris Ser. subsp. siceraria. & Cucurbitaceae & Vishapaha & M.P.N.7/11 \\
\hline 2. & Vandhya karkotaki & Momordica cochichinensis Spr. & Cucurbitaceae & Vishajith & M.P.N.7/30 \\
\hline 3. & Vishamusti & Cucumis melo Linn. & Cucurbitaceae & Vishapaha & M.P.N.7/31 \\
\hline 4. & Loni & Portulaca quadrifolia Linn. (loni) & Portulaceaea & Vishapaha & M.P.N.7/53 \\
\hline 5. & Kasamarda & Cassia occidentalis Linn. & Caesalpinaceae & Vishajith & M.P.N.7/64 \\
\hline 6. & Karira & Capparis decidua (Forssk.) Edgew. & Capparidaceae & Visha & M.P.N.7/70 \\
\hline
\end{tabular}

\section{Paniyadi Varga ${ }^{[5]}$}

Jala, Ganga jala (Akasa jala), Chandrakantha jala in Jala varga; Gritha in Dugda varga; Taila, Simsapa taila, Agaru taila, Gandira taila, Rasala taila, Devadaru taila in Taila varga; Madhvasava in Madhya varga; Mutra (general), Hasthi mutra, Aja mutra, Nara mutra in mutra varga are vishahara. Among this Taila has specific indication in Vyala visha and Naramutra in Gara visha.

\section{Dhanyaguna varga ${ }^{[6]}$}

Shali dhanya (raktasali), Maha shali, Vana kulattha, Adhaki, Kusumbha, Kodrava are vishahara.

\section{Mamsa varga ${ }^{[7]}$}

Sarpa mamsa has a specific indication in Dushivisha

\section{DISCUSSION}

After a thorough review of Madanapala Nighantu, 105 dravyas are vishahara in nature. 21 dravyas in Abhayadai varga (Table no.1), 1 dravya in Sunthyadi varga (Table no.2), 22 dravyas in Karpuradi varga (Table no.3), 18 dravyas in Suvarnadi varga (Table no.4), 12 dravyas in Vatadi varga (Table no.5), 3 dravyas in Phaladi varga (Table no.6), 6 dravyas in Sakadi varga (Table no.7), 15 dravyas in Paniyadi varga), 6 dravyas in Dhanya varga, 1 dravya in Mamsa varga are having vishahara property. The last chapter of Madanapala Nighantu is Misraga varga which deals with certain miscellaneous topics such as Anupana, Dinacharya, Ritucharya, Sodhana, Shatrasa etc., in which he explains Uttara basti is good for visharogi), Katu rasa is vishahara, likewise, if Vamana procedure is carried out visharoga will not reoccur. ${ }^{[8]}$ Among these 105 dravyas majority of the dravyas have Tikta, Katu, Kashaya and madhura rasa (not predominant as the other 3 rasas). Most of them are having Laghu, Ruksha, Tikshna guna, the majority with Katu vipaka and in the case of Virya, Ushna and Seeta virya goes hand in hand.

According to Ashtanga hridaya, when Visha enters the body, it first vitiates rakta, then kapha, pitta and anila along with their ashaya and when enters hridaya it leads to marana. ${ }^{[9]}$ While considering rasa; Katu, Tikta, Kashaya rasa pacifies kapha dosa. Kashaya, Tikta, Madhura rasa pacifies pitta dosa and Madhura rasa pacifies vata dosa ${ }^{[10]}$ Meanwhile Tikta rasa and Katu rasa are vishahara in nature according to Charaka samhitha and Madanapala Nighantu respectively. ${ }^{[11][12]}$ While considering the guna; Laghu, Ruksha and Tikshna gunas helps in the easy and fast penetration of dravyas to provide faster action on visha. The Katu vipaka will pacify Kapha dosa. [13] Seeta virya pacifies pitta dosa, it also pacifies rakta as pitta dosa have ashraya sthana on rakta. [14][15] Ushna virya pacifies kapha and vata dosas. ${ }^{[14]}$ After all, these dravyas are vishahara in karma, so that they have the ability to protect the sarira from visha by all means.

This article is a thorough compilation of all the vishahara dravyas explained in the Madanapala Nighantu with their botanical name and family, so as to provide easy identification of the dravyas. Also included are the indications of these dravyas and some of the dravyas are having specific indications to certain visha which are not known. The dravyas mentioned here can be either used as a single drug formulation or can be used in combinations for various poisoning. While going through this article it is clear that not only mere drugs but also other substances such as jala, dugda, dhanya and even some sodhana procedures are explained in Madanapala Nighantu as vishahara. It says the importance of vishahara dravyas as pathya and charya, thus providing a base for the researcher to do further research on the dravyas 
mentioned in the Madanapala Nighantu by keeping this article as a reference.

\section{CONCLUSION}

From the above discussion, it can be concluded that 105 dravyas in Madanapala Nighantu are having vishahara property. Among that some of the dravyas are having specific indications. So, it is clear that either the single drug preparation or their combinations can give great results in the management of various poisoning. So, more studies on these dravyas are required to establish the vishahara property and thus help to open up new scopes in the management of visha.

\section{REFERENCES}

1. K Shobha bhat, A Textbook of Agadatantra. Chapter1, Varanasi: Chaukamba Orientalia,2018: 2

2. Acharya J T, editor, Commentary Nibandha sangraha of Dalhanaacharya on Susrutha Samhitha of Susrutha, Kalpa sthana; Jangama visha vijnaniyam kalpam: Chapter 3, Verse 21. Varanasi: Chaukambha Sanskrit Sansthan,2017; 539

3. Www.wisdomlib.org. Nighantu [Internet]. Wisdom Library; The portal for Hinduism, Sanskrit, Buddhism, Jainism, Mesopotamia etc... 2021 [cited 2021Oct2]. Available https://www.wisdomlib.org/definition/nighantu

4. Sastry J L N, English translation on Madanapala Nighantu. Varanasi: Chaukhamba Orientalia, 2010

5. Sastry J L N, English translation on Madanapala Nighantu. Paniyadi varga: Chapter 8, Verse 3,8,19,128,139,152,184,210,211,213, 217. Varanasi: Chaukhamba Orientalia, 2010: 715-8, 739,743,745,752,758-9

6. Sastry J L N, English translation on Madanapala Nighantu. Dhanyaguna varga: Chapter10, Verse 9,46,52,54,64. Varanasi: Chaukhamba Orientalia, 2010: 774,792-3,797,803

7. Sastry J L N, English translation on Madanapala Nighantu. Mamsa varga: Chapter12, Verse 108.Varanasi: Chaukhamba Orientalia, 2010: 936

8. Sastry J L N, English translation on Madanapala Nighantu. Misraga varga: Chapter13, Verse 64,68,74,75. Varanasi: Chaukhamba Orientalia, 2010: $957-8$

9. Vaidya Bhisagacharya Harishastri Paradkar, editor, Commentary Sarvangasundari of Arunadatta and Ayurvedarasayana of Hemadri on Ashtangahrdayam of Vagbhata, Uttarasthana; Vishaprathishedha: Chap- ter 35, Verse 10. Varanasi: Chaukambha Krishnadas Academy.903

10. Vaidya Bhisagacharya Harishastri Paradkar, editor, Commentary Sarvangasundari of Arunadatta and Ayurvedarasayana of Hemadri on Ashtangahrdayam of Vagbhata, Sutrasthana; Ayushkamiyam: Chapter1, Verse 15. Varanasi: Chaukambha Krishnadas Academy.11

11. Acharya Vidhyadhar Shukla, Ravi Dutt Tripathi, Vaidyamanorama Hindi commentary on Charaka samhitha of Agnivesa, Sutrasthana; Athreyabadhrakapiyam adhyaya: Chapter 26, Verse45. Delhi: Chaukamba Sanskrit Pratisthan.379

12. Sastry J L N, English translation on Madanapala Nighantu. Misraga varga: Chapter13, Verse 68. Varanasi: Chaukhamba Orientalia, 2010: 958

13. L Hegde Prakash, A Harini, A Textbook of Dravyaguna Vijnana. Vipaka parjnana: Chapter 6. Delhi: Chaukhamba publication,2011; Vol 1: 277

14. L Hegde Prakash, A Harini, A Textbook of Dravyaguna Vijnana. Virya parjnana: Chapter 7. Delhi: Chaukhamba publication,2011; Vol 1: 308,309

15. Vaidya Bhisagacharya Harishastri Paradkar, editor, Commentary Sarvangasundari of Arunadatta and Ayurvedarasayana of Hemadri on Ashtangahrdayam of Vagbhata, Sutrasthana; Dosadivijnaniyam: Chapter11, Verse26,27. Varanasi: Chaukambha Krishnadas Academy.186

\section{Source of Support: Nil \\ Conflict of Interest: None Declared}

How to cite this URL: Haritha M et al: Vishahara Dravyas In Madanapala Nighantu - A Review Article. International Ayurvedic Medical Journal \{online\} 2021 \{cited December 2021\} Available from: http://www.iamj.in/posts/images/upload/3005 3011.pdf 